\title{
Fault Detection in Base-Isolation Systems via a Restoring Force Observer
}

\author{
Yolanda Vidal, Leonardo Acho, Francesc Pozo and José Rodellar
}

\begin{abstract}
This paper proposes a fault detection method for hysteretic base-isolation systems. One of the key contributions of this work is a Lyapunov-based restoring force observer that leads to the design of a robust fault detection scheme. The different fault types considered are stiffness and damping variations in the system. The proposed fault estimation method provides a direct estimate of the size and severity of the fault, which can be important in many civil engineering applications. A design procedure is described, and nonlinear simulation results are presented to demonstrate the applicability of the proposed method.
\end{abstract}

\section{INTRODUCTION}

Base isolation is a collection of structural elements of a building that should substantially decouple the building's structure from the shaking ground; thus, they protect the building's integrity and enhance its seismic performance. Base isolation tends to restrict transmission of the ground motion to the building, and it also keeps the building positioned properly over the foundation. For example, sliding and elastomeric bearing systems reduce the building response to seismic excitation, but with increased base displacements in near-fault motions. The current practice is to provide nonlinear passive dampers to limit the bearing displacements. However, this increases the forces in the superstructure and at the isolation level. Active and semiactive control using novel devices, such as magnetorheological (MR) dampers, present attractive alternatives to passive nonlinear devices (see [1], [2], [3], [4]). In this work, a passive second-order, base-isolated system is used for simplicity; however, it is straightforward to generalize the obtained results to active and semiactive control.

In nonlinear control theory, fault detection has attracted significant interest as can be seen in the works of [5], [6], [7], [8], and [9]. Moreover, when operating highly reliable systems, the primary interest is to detect a fault at the earliest possible stage (see [10]). A system that continuously monitors a structure to detect damage is often referred to as a health monitoring system in the mechanical, aerospace, and civil engineering fields. A fault detection technique detects faults by means of a residual signal (see [11]) produced by available measurements. It must be a signal that is close to zero in the absence of a fault, and significantly affected in the presence of faults (see [10], [11], and [12]). The main components of a fault detection system are the following: a residual generator signal, residual evaluation, and the

CoDAlab, Departament de Matemàtica Aplicada III, Escola Universitària d'Enginyeria Tècnica Industrial de Barcelona, Universitat Politècnica de Catalunya, Comte d'Urgell, 187, 08036 Barcelona, Spain. http: / / www-ma3.upc.es / codalab decision-making process. In addition, the residual signal has to return to its original no-fault detection stage when the fault vanishes. Various methods have been proposed, among which the observer-based fault-detection techniques have yielded the best results (see [6], [7], and [8]). The basic idea behind the observer-based approaches is to estimate the outputs of the system from the measurements by using some type of observer, and then construct the residual by a properly weighted output estimate error. This paper proposes a fault detection method following the observer-based approach. The different fault types considered are stiffness and damping variations in the system. A residual signal is obtained that can be examined for the likelihood of faults in hysteretic baseisolator devices. As expected, the residual signal returns to its original no-fault detection stage when the fault vanishes, but also provides a direct estimate of the size and severity of the fault, which can be important in many applications.

The paper is structured as follows. The problem statement is presented in Section II. Next, the fault detection method is developed in detail in Section III and different fault types considered in this paper are presented. To illustrate the efficiency of the proposed method, numerical simulations are analyzed for hysteretic structural systems in the presence of seismic excitations (the recorded earthquake El Centro is used as in [13]) in Section IV. Finally, the conclusions and future work are stated in Section V.

\section{PROBLEM STATEMENT}

Consider a passive second-order base-isolated structure (see [14]) given by

$$
m \ddot{x}+c \dot{x}+\Phi(x, t)=f(t)
$$

where $m$ and $c$ are the mass and the damping coefficients, respectively; $\Phi(x, t)$ characterizes a nonlinear restoring force, where $x$ gives the position and $f(t)$ is an exciting but bounded unknown force given by the earthquake ground acceleration. The nonlinear force $\Phi(x, t)$ describes a hysteresis behavior. It can be due to the presence of passive inelastic rubber bearings, other passive isolation devices [15], semiactive MR dampers [16] or other hysteretic control devices, and it can be described by the so-called Bouc-Wen model as in [17] in the following form:

$$
\begin{aligned}
\Phi(x, t) & =\alpha_{0} \kappa x(t)+\left(1-\alpha_{0}\right) D \kappa \omega(t), \\
\dot{\omega} & =D^{-1}\left(A \dot{x}-\beta_{0}|\dot{x}||\omega|^{n-1} \omega-\lambda \dot{x}|\omega|^{n}\right)+\Delta(t) .
\end{aligned}
$$


This model represents the restoring force $\Phi(x, t)$ by the superposition of an elastic component $\alpha_{0} \kappa x(t)$ and a hysteresis component $\left(1-\alpha_{0}\right) D \kappa \omega(t)$, in which $D>0$ is the yield constant displacement and $\alpha_{0} \in(0,1)$ is the post- to pre-yielding stiffness ratio. The hysteretic component involves a non-dimensional auxiliary variable, $\omega(t)$, which is the solution of the nonlinear differential equation (3). In equation (1), $A, \beta$ and $\lambda$ are nondimensional parameters that control the shape and size of the transition from the elastic to plastic response (see [17] and [18]). Finally, the term $\Delta(t)$ in equation (3) represents an unknown fault in the MR damper system. A detailed discussion of this term is given in the next section; however, broadly speaking, when $\Delta(t)=0$ the system is healthy and otherwise a fault has occurred.

Because state variables describe the state evolution of the dynamical system (see [19]), any change in the system's dynamic properties will be registered by the state variables. The Bouc-Wen model has only one state variable; thus, any change (fault) in its dynamic behavior will be registered. As a result, the fault term, $\Delta(t)$, is added to the dynamic of the internal variable. Recall that the fault detection method assumes that the hysteretic base-isolation is represented by the Bouc-Wen model. This model has gained large consensus within the engineering community because it can capture a wide variety of different shapes of the hysteresis loops as can be seen in [20]. Although the internal parameters of the Bouc-Wen model can be manipulated by applying a voltage in magnetorheological dampers, for simplicity, we assume that they are constant as in [14]. Moreover, many baseisolated structures have hysteretic behavior with constant parameters as can be seen in [21].

The objective of this paper is to detect a failure in the base-isolated structure, which is equivalent to finding a fault in $\omega(t)$. It is noteworthy that the internal variable $\omega(t)$ is uniformly bounded for any piecewise discontinuous signal $\dot{x}(t)$, for certain values of the parameters $A, \beta$ and $\lambda$. Theorem 1 in [14] proves this statement and provides a way to compute the bound.

The state representation of (1)-(3) yields

$$
\begin{aligned}
\dot{x} & =y, \\
\dot{y} & =\frac{f(t)}{m}-\frac{c}{m} y-\frac{\Phi(x, t)}{m}, \\
\dot{\omega} & =D^{-1}\left(A \dot{x}-\beta_{0}|\dot{x}||\omega|^{n-1} \omega-\lambda \dot{x}|\omega|^{n}\right)+\Delta(t) .
\end{aligned}
$$

The main goal of this paper is to design a residual signal that is able to detect the presence of the unknown fault $\Delta(t)$. This residual signal must be close to zero when $\Delta(t)=0$, and otherwise, it must be far from zero. In order to design the fault detection method, let us assume that the following conditions are satisfied:

- position $(x)$, velocity $(\dot{x})$, and seismic perturbation $(f(t))$ are measurable signals.

- the nominal parameters in (4)-(6) are known.

- the nonlinear restoring force, $\Phi(x, t)$, is unknown.

Note that because of the unknown restoring force, $\Phi(x, t)$, an observer is necessary to fulfill the objective.

\section{FAULT DETECTION METHOD}

The aim of this section is to model the various subsystems of the fault detection method (see Fig. 1), namely

- a real plant, modeled in this paper via (4)-(6), and from which position $(x)$, velocity $(\dot{x})$, and seismic perturbation $(f(t))$ are measurable signals;

- a restoring force observer that uses the measured signals $x, \dot{x}$, and $f(t)$ to construct an observer $\hat{\Phi}(x, t)$ of the unknown $\Phi(x, t)$;

- a healthy model, to simulate the behavior of the system in the absence of a fault;

- a residual signal generator.

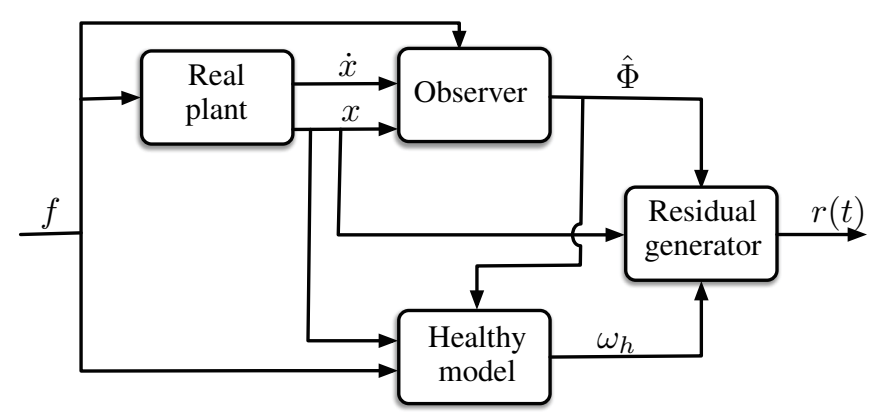

Fig. 1. Block diagram of the fault detection method.

\section{A. Real Plant}

The real plant is modeled using equations (4)-(6). Recall that position $(x)$, velocity $(\dot{x})$, and seismic perturbation $(f(t))$ are measurable signals. Two types of faults in the baseisolation system are modeled: changes in the stiffness and changes in the damping of the device.

Faults due to a change in the stiffness of the base-isolation system (caused, for example, by the leakage of the MR damper's fluid) are modeled by adding an additional term, $\Delta A$, to the nominal value of $A$. That is, when a fault in the stiffness is present, the internal variable dynamic is modeled as

$$
\dot{\omega}=D^{-1}\left((A+\Delta A) \dot{x}-\beta_{0}|\dot{x}||\omega|^{n-1} \omega-\lambda \dot{x}|\omega|^{n}\right),
$$

that can be written as

$$
\dot{\omega}=D^{-1}\left(A \dot{x}-\beta_{0}|\dot{x}||\omega|^{n-1} \omega-\lambda \dot{x}|\omega|^{n}\right)+D^{-1} \Delta A \dot{x} .
$$

Recall that the restoring force $\Phi(x, t)$, given in equation (2), depends on $\omega$. Therefore, the effect of the fault on $\Phi(x, t)$ can be obtained by integrating the added term $D^{-1} \Delta A \dot{x}$, which gives $D^{-1} \Delta A x$. That is, an additional stiffness term is added to the restoring force to simulate a fault in the stiffness of the base-isolation system.

Following the same idea, faults due to a change in the damping of the base-isolation system are modeled by adding an additional term, $D^{-1} \Delta A \ddot{x}$, to the dynamic of the internal variable. That is, when a fault in the damping is present, the real plant is modeled as

$$
\dot{\omega}=D^{-1}\left(A \dot{x}-\beta_{0}|\dot{x}||\omega|^{n-1} \omega-\lambda \dot{x}|\omega|^{n}\right)+D^{-1} \Delta A \ddot{x} .
$$


The effect of the fault on $\Phi(x, t)$ can be obtained by integrating the added term $D^{-1} \Delta A \ddot{x}$, which gives $D^{-1} \Delta A \dot{x}$. In other words, a damping term is added to the restoring force, which simulates a fault in the damping of the baseisolation system.

\section{B. Restoring Force Observer Design}

In this section, an observer, $\hat{\Phi}(x, t)$, of the restorting force $\Phi(x, t)$ is presented.

The observer assumes that position $(x)$, velocity $(\dot{x})$, and seismic perturbation $(f(t))$ can be measured. In addition, since there is no prior information about the derivative of the restoring force, it is reasonable to suppose that $\dot{\Phi}=0$, which implies that the restoring force varies slowly relative to the observer dynamics. In fact, in [22], [23] it is shown, by simulation and experiment, that an observer designed under the previous assumption can also track some fast timevarying disturbances. Thus, the hypothesis $\dot{\Phi}=0$ is not a very restrictive assumption.

Theorem 1: Consider the system

$$
\begin{aligned}
& \dot{\hat{y}}=\left(k_{1}(\dot{x}-\hat{y})-c \dot{x}+f-\hat{\Phi}\right) / m, \quad k_{1}>0, \\
& \dot{z}=-k_{2} z+\hat{y}-\left(k_{2} c-k_{2}^{2} m\right) \dot{x}+k_{2} x+k_{2} f, \quad k_{2}>0,
\end{aligned}
$$

where $k_{1}, k_{2}$ are design parameters and

$$
\hat{\Phi}=z-x-k_{2} m \dot{x} .
$$

If $\dot{\Phi}=0$, then $\hat{\Phi}$ tends to $\Phi$ as time goes to infinity. If $\dot{\Phi} \neq 0$, the error $e=\Phi-\hat{\Phi}$ is ultimately bounded.

Proof: Consider the Lyapunov function

$$
V_{1}=\frac{1}{2}(\Phi-\hat{\Phi})^{2}+\frac{m}{2}(\dot{x}-\hat{y})^{2} .
$$

Differentiating the positive definite function $V_{1}$ along the system trajectory, and taking into account that $\dot{\Phi}=0$, yields

$$
\dot{V}_{1}=(\Phi-\hat{\Phi})(-\dot{\hat{\Phi}}-\dot{x}+\hat{y})-k_{1}(\dot{x}-\hat{y})^{2} .
$$

Clearly, taking

$$
\dot{\hat{\Phi}}=-\dot{x}+\hat{y}+k_{2}(\Phi-\hat{\Phi})
$$

then

$$
\dot{V}_{1}=-k_{2}(\Phi-\hat{\Phi})^{2}-k_{1}(\dot{x}-\hat{y})^{2},
$$

and, thus, $\dot{V}_{1}$ is negative semi-definite. To complete the proof it only remains to see that the equation (12) corresponds to equations (10) and (11). For this purpose, replace $\Phi$ in (12) by the equation (1) to obtain

$$
\dot{\hat{\Phi}}=-\dot{x}+\hat{y}+k_{2}(-m \ddot{x}-c \dot{x}+f-\hat{\Phi}),
$$

and arranging terms yields

$$
k_{2} m \ddot{x}+\dot{x}+\dot{\hat{\Phi}}=\hat{y}+k_{2}(-c \dot{x}+f-\hat{\Phi}) .
$$

Defining the right hand side of the previous equation as $\dot{z}$ and integrating leads to

$$
\hat{\Phi}=z-x-k_{2} m \dot{x} .
$$

Notice that using the previous equation, $\dot{z}$ can be written as

$$
\dot{z}=-k_{2} z+\hat{y}-\left(k_{2} c-k_{2}^{2} m\right) \dot{x}+k_{2} x+k_{2} f .
$$

\section{Healthy Model Observer}

The healthy model is developed in order to simulate the behavior of the system in the absence of a fault. The system is modeled as

$$
\begin{aligned}
\dot{x}_{h} & =y_{h}, \\
\dot{y}_{h} & =\frac{f}{m}-\frac{c}{m} y_{h}-\frac{\Phi_{h}}{m}+v \\
\Phi_{h} & =\alpha_{0} \kappa x_{h}+\left(1-\alpha_{0}\right) D \kappa \omega_{h}(t) \\
\dot{\omega}_{h} & =D^{-1}\left(A \dot{x}_{h}-\beta_{0}\left|\dot{x}_{h}\right|\left|\omega_{h}\right|^{n-1} \omega_{h}-\lambda \dot{x}_{h}|\omega|_{h}^{n}\right),
\end{aligned}
$$

where $v$ is a control law to be chosen in order to ensure that $e_{1}:=x-x_{h}$ and $e_{2}:=y-y_{h}$ are uniformly ultimately bounded. Recall that $e_{3}:=\omega-\omega_{h}$ is bounded because both variables are internal variables of a Bouc-Wen model, and thus, they are already bounded.

In other words, the system in equations (13)-(16) is an observer of the system in equations (1)-(6) and converges to it if $\Delta(t)=0$. Otherwise, the system in equations (13)-(16) will detect the failure in the system (1)-(6), and that is why we call the system (13)-(16) a healthy model observer.

Loosely speaking, we would like to find state feedback control for the system (13)-(16) that guarantees that every response of the system is uniformly ultimately bounded within a set containing the zero state. Let's take the Lyapunov function $V_{2}=\frac{\alpha_{0} \kappa}{2 m} e_{1}^{2}+\frac{1}{2} e_{2}^{2}$, then the derivative of $V_{2}$ along the system trajectory yields

$$
\begin{aligned}
\dot{V}_{2} & =\frac{\alpha_{0} \kappa}{m} e_{1} \dot{e_{1}}+e_{2} \dot{e_{2}}=\frac{\alpha_{0} \kappa}{m} e_{1}\left(\dot{x}-\dot{x_{h}}\right)+e_{2}\left(\dot{y}-\dot{y}_{h}\right) \\
& =\frac{\alpha_{0} \kappa}{m} e_{1} e_{2}+e_{2}\left[-\frac{c}{m}\left(y-y_{h}\right)-\frac{\Phi-\hat{\Phi}+\hat{\Phi}-\Phi_{h}}{m}-v\right] \\
& =\frac{\alpha_{0} \kappa}{m} e_{1} e_{2}-\frac{c}{m} e_{2}^{2}-(\Phi-\hat{\Phi}) \frac{e_{2}}{m}-\left(\hat{\Phi}-\Phi_{h}\right) \frac{e_{2}}{m}-v e_{2} .
\end{aligned}
$$

Taking $v=\frac{\alpha_{0} \kappa}{m} e_{1}-\frac{\left(\hat{\Phi}-\Phi_{h}\right)}{m}$,

$$
\dot{V}_{2}=(\hat{\Phi}-\Phi) \frac{e_{2}}{m}-\frac{c}{m} e_{2}^{2},
$$

and therefore, $\dot{V}_{2} \leq 0$ when $\left|e_{2}\right| \geq \frac{|\Phi-\hat{\Phi}|}{c}$. It can be concluded that the solution is uniformly ultimately bounded with the ultimate bound

$$
\left|e_{2}\right|<\frac{|\Phi-\hat{\Phi}|}{c} .
$$

Note that the size of the ultimate bound depends on the performance of the observer. As the performance of $\hat{\Phi}$ improves, the ultimate bound decreases.

To summarize, our healthy model (13)-(16) is completed using the control law

$$
v=\frac{\alpha_{0} \kappa}{m} e_{1}-\frac{\left(\hat{\Phi}-\Phi_{h}\right)}{m} .
$$




\section{Residual Signal Design}

In the field of fault detection and identification, a residual signal should be defined to detect and identify fault signals. Note that using the measurement of $x$ and the observer $\hat{\Phi}$, the variable $\omega$ can be estimated from equation (2) obtaining,

$$
\hat{\omega}=\frac{\hat{\Phi}-\alpha_{0} \kappa x}{\left(1-\alpha_{0}\right) D \kappa}
$$

A first trial for the residual signal was a weighted function of the difference between $\hat{\omega}$ and $\omega_{h}$. However, this residual signal would stabilize after the fault vanishes, but it would not return to zero. This is because the internal variable of the Bouc-Wen model does not return to zero after the earthquake (or after a fault) passes away but rather stabilizes to a different value (because of the memory effect of hysteresis). In order to circumvent this issue, the following residual signal is used

$$
r(t)=K\left(\dot{\hat{\omega}}-\dot{\omega}_{h}\right)
$$

where $K$ is the weight, and $\dot{\hat{\omega}}$ is obtained by numerical differentiation of $\hat{\omega}$. Several numerical differentiation procedures can be used. Here, differentiation of the cubic spline approximation is used.

When the residual signal is close to zero, the system is healthy, otherwise the residual signal not only indicates the presence of a fault but also gives a measure of the severity of that fault.

\section{NUMERICAL SIMULATION}

In order to investigate the efficiency of the proposed health monitoring scheme, the El Centro earthquake is used (see Fig. 2). The parameters used in the simulations have the following nominal values: $m=156 \times 10^{3} \mathrm{Kg}, c=2 \times$ $10^{4} \mathrm{Ns} / \mathrm{m}, \kappa=6 \times 10^{6} \mathrm{~N} / \mathrm{m}, \alpha_{0}=0.6, D=0.6 \mathrm{~m}, A=$ $1, \beta_{0}=0.1, \lambda=0.5$, and $n=3$ as in [14] and [24].

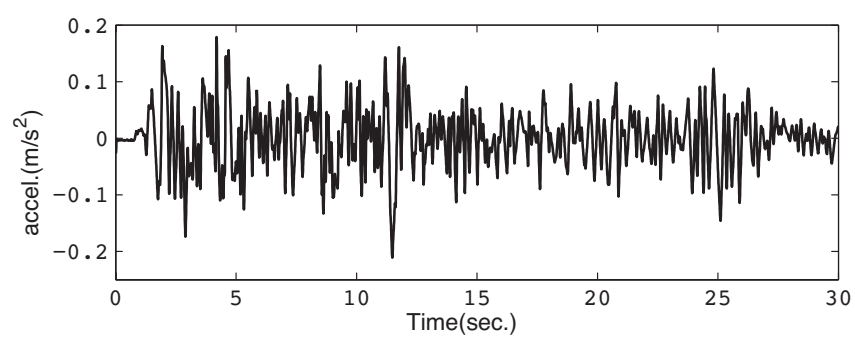

Fig. 2. El Centro earthquake, ground acceleration.

\section{A. Stiffness and Damping Faults}

Design parameters $k_{1}$ and $k_{2}$ in (9) and (10) are set equal to 400 , and the constant $K$ in (17) is set equal to 45. The parameter $\Delta A$ in equation (7) varies with time, thus simulating a stiffness fault in the base-isolated system. Simulation results are shown in Fig. 3.

The first row in Fig. 3 shows the real restoring force $\Phi$ versus the observed one $\hat{\Phi}$ (left) and the absolute error among these quantities (right). The second row shows $\hat{\omega}$ versus the internal variable given by the healthy model, $\omega_{h}$, (left) and the absolute error among these quantities (right). Note that, when the fault vanishes, the absolute error $\hat{\omega}-\omega_{h}$ stabilizes to a constant value that is usually not zero. This is because the internal variable of the Bouc-Wen model does not return to zero after the earthquake (or after a fault) passes away but stabilizes to a different value (because of the memory effect of hysteresis). The third row in Fig. 3 shows the residual signal (left) and the faulty increment, $\Delta A$, used in the simulations (right). As desired, the residual signal is close to zero in the absence of a fault and is significantly affected in the presence of faults. As expected, the residual signal returns to its original no-fault detection stage (close to zero) when the fault vanishes. However, small variations in the system stiffness $(\Delta A=-0.2)$ are not clearly detected by the fault detection method. Further work should be done to improve the sensitivity of the method.

In order to model the damping fault, the parameter $\Delta A$ in equation (8) varies with time and, therefore, simulates a damping fault in the base-isolated system. Simulation results are shown in Fig. 4.

Note that the observer, $\hat{\Phi}$, is a different approximation of $\Phi$ than the one obtained in the stiffness fault simulation. This is because the observer uses the real plant measurements of position, velocity and force that are affected by the fault. As different faults are simulated, different approximations are obtained. Again, when the fault vanishes $(\Delta A=0)$, the absolute error $\hat{\omega}-\omega_{h}$ stabilizes to a constant value that is usually not zero, and the residual signal is close to zero in the absence of a fault and is significantly affected in the presence of faults.

\section{CONCLUSIONS AND FUTURE WORKS}

\section{A. Conclusions}

This paper proposes a novel Lyapunov-based restoring force observer that allows the design of a robust fault detection method. The fault detection goal is fulfilled because a residual signal is designed that is close to zero in the absence of a fault and is significantly affected in the presence of a fault. As expected, the residual signal returns to its original no-fault detection stage when the fault vanishes, but also provides a direct estimate of the size and severity of the fault, which can be important in many civil engineering applications.

\section{B. Future Works}

As future work it remains to examine the robustness of the proposed algorithm when noise is present in the measured signals and the robustness with respect to parametric uncertainty. It also would be interesting to apply the proposed fault detection method to the benchmark problem for seismically excited base-isolated buildings (multiple degrees of freedom problem) proposed by [13]. Finally, it is important to test the scheme in an experimental setup with a shacking table and a real MR-damper which will easily allow to modify the damping behavior, thus simulating a fault. This experimental 

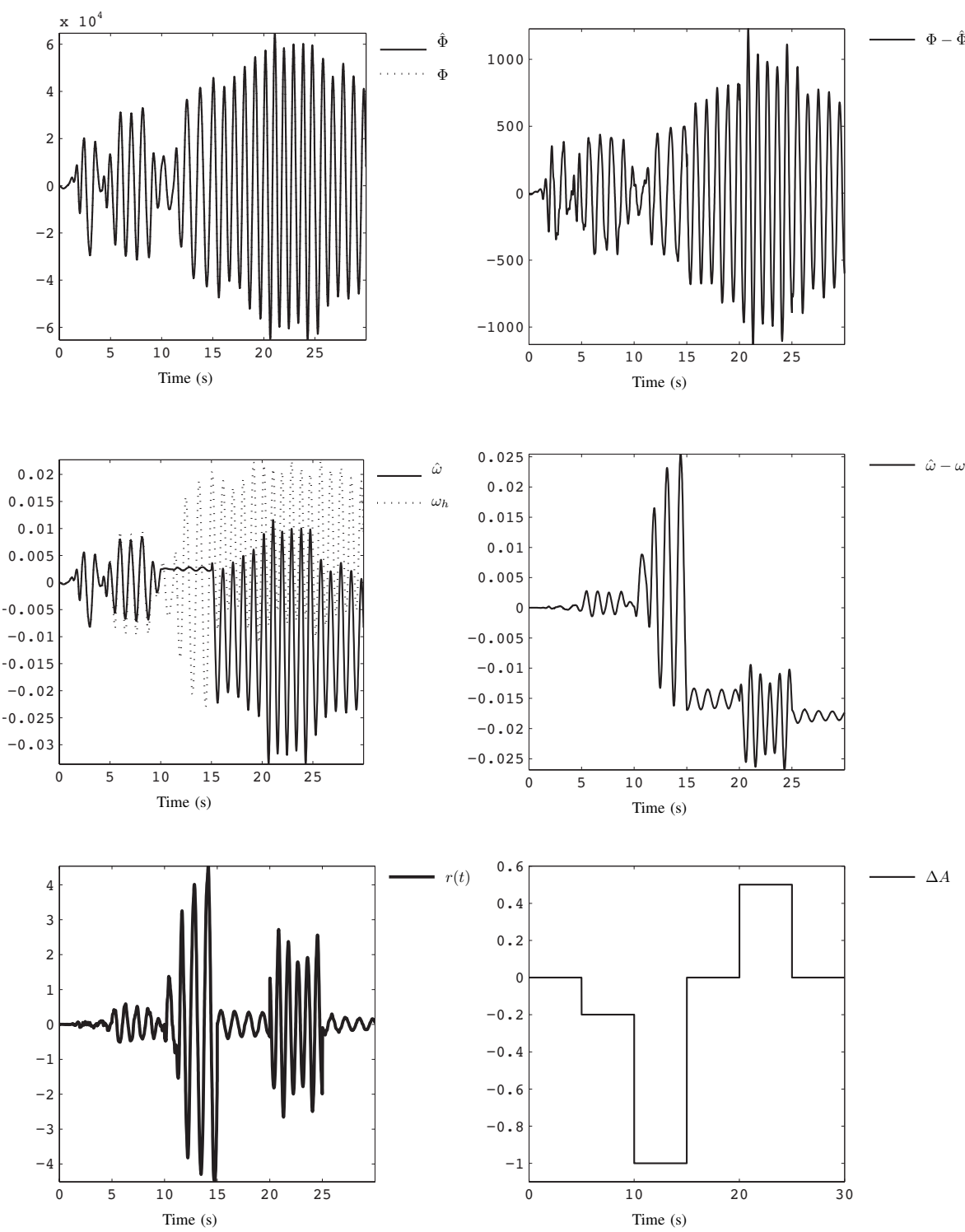

Fig. 3. Stiffness fault present in the system.

setup will also be helpful to give the minimal size of the fault that can be detected by the proposed technique.

\section{ACKNOWLEDGMENTS}

This work was supported in part by CICYT (Spanish Ministry of Science and Innovation) by funds DPI200806463-C02-01 and CIT-020000-2008-40.

\section{REFERENCES}

[1] B. F. Spencer Jr and S. Nagarajaiah, "State of the art of structural control," Journal of Structural Engineering ASCE, vol. 129, no. 7, pp. 845-856, 2003.

[2] F. Pozo, P. M. Montserrat, J. Rodellar, and L. Acho, "Robust active control of hysteretic base-isolated structures: Application to the benchmark smart base-isolated building," Structural Control and Health Monitoring, vol. 15, no. 5, pp. 720-736, 2008.

[3] F. Pozo, L. Acho, and J. Rodellar, "Hyperbolic control for vibration mitigation of a base-isolated benchmark structure," Structural Control and Health Monitoring, Accepted.
[4] M. Zapateiro, H. R. Karimi, N. Luo, and B. F. Spencer Jr, "Realtime hybrid testing of semiactive control strategies for vibration reduction in a structure with mr damper," Structural Control and Health Monitoring, Published online.

[5] R. Patton, "Robustness in model-based fault diagnosis: the 1997 situation," IFAC Annual Reviews, vol. 21, pp. 101-121, 1997.

[6] G. R. Duan and R. J. Patton, "Robust fault detection in linear systems using Luenberger observers," in UKACC International Conference on Control 98, Vols I and II. Inst Electrical Engineers Inspec Inc, 1998, Proceedings Paper, pp. 1468-1473, Swansea, Wales.

[7] C. Edwards, S. K. Spurgeon, and R. J. Patton, "Sliding mode observers for fault detection and isolation," Automatica, vol. 36, no. 4, pp. 541553, 2000.

[8] M. Saif, "Fault diagnosis based on equivalent control concept," in Robotics, Automation and Control and Manufacturing: Trends, Principles and Applications, ser. TSI PRESS SERIES, vol. 14, 2002, Proceedings Paper, pp. 423-428, 5th Biannual World Automation Congress, ORLANDO, FL, USA.

[9] P. Mhaskar, C. McFall, A. Gani, P. D. Christofides, and J. F. Davis, "Isolation and handling of actuator faults in nonlinear systems," Automatica, vol. 44, no. 1, pp. 53-62, 2008.

[10] S. Liberatore, J. L. Speyer, and A. C. Hsu, "Application of a fault 

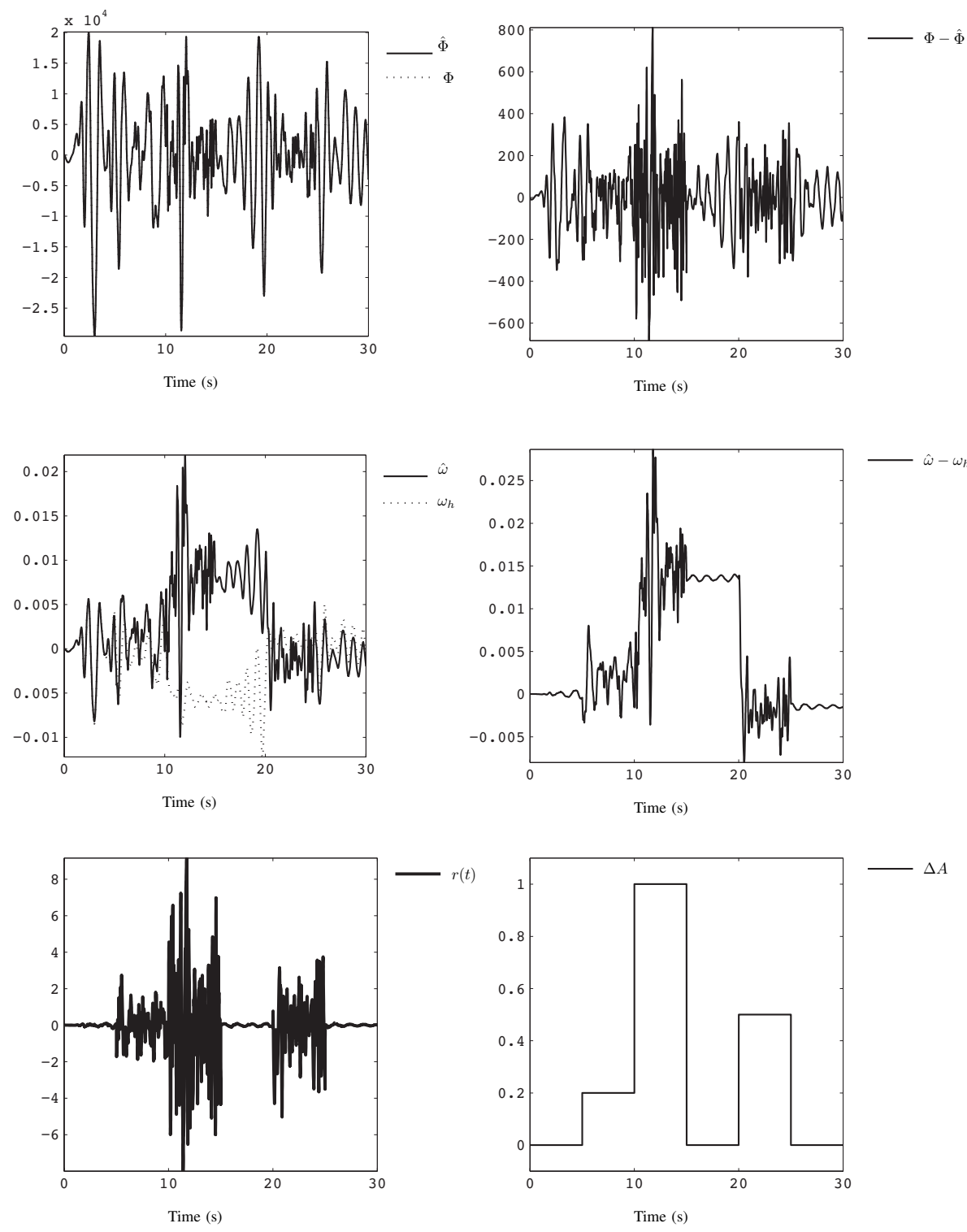

Fig. 4. Damping fault present in the system.

detection filter to structural health monitoring," Automatica, vol. 42 , pp. 1199-1209, 2006.

[11] G. Besanon, "High-gain observation with disturbances attenuation and application to robust fault detection," Automatica, vol. 39, pp. 1095$1102,2003$.

[12] M. Kinnaert, "Robust fault detection based on observers for bilinear systems," Automatica, vol. 35, pp. 1829-1842, 1999.

[13] S. Narasimhan, S. Nagarajaiah, E. A. Johnson, and H. P. Gavin, "Smart base-isolated benchmark building. part i: Problem definition," Structural Control and Health Monitoring, vol. 13, pp. 573-588, 2006.

[14] F. Ikhouane, V. Manosa, and J. Rodellar, "Adaptive control of a hysteretic structural system," Automatica, vol. 41, no. 2, pp. 225-231, 2005.

[15] M. Ismail, J. Rodellar, and I. F, "An innovative isolation device for aseismic design," Engineering Structures, vol. 32, no. 4, pp. 1168$1183,2010$.

[16] F. Ikhouane and J. Rodellar, Systems with Hysteresis: Analysis, Identification and Control Using the Bouc-Wen Model. UK: John Wiley \& Sons Ltd, 2007.

[17] _ "On the hysteretic Bouc-Wen model," Nonlinear Dynamics, vol. 42, no. 1, pp. 63-78, 2005.

[18] A. W. Smyth, S. F. Masri, E. B. Kosmatopoulos, A. G. Chassiakos, and T. K. Caughey, "Development of adaptive modeling techniques for non-linear hysteretic systems," International Journal of Non-Linear Mechanics, vol. 37, no. 8, pp. 1435-1451, 2002.

[19] H. K. Khalil, Nonlinear Systems. New Jersey, USA: Prentice-Hall, 2002 (Third edition).

[20] F. Ma, H. Zhang, A. Bockstedte, G. C. Foliente, and P. Paevere, "Parameter analysis of the differential model of hysteresis," Transactions of the ASME, vol. 71, pp. 342-349, 2004.

[21] M. Ismail, F. Ikhouane, and J. Rodellar, "The Hysteresis Bouc-Wen Model, a Survey," Archives of Computational Methods in Engineering, vol. 16, no. 2, pp. 161-188, 2009.

[22] W. H. Chen, D. J. Ballance, P. J. Gawthrop, and J. O'Reilly, "A nonlinear disturbance observer for robotic manipulators," IEEE Transactions on Industrial Electronics, vol. 47, no. 4, pp. 932-938, 2000.

[23] W. H. Chen, D. J. Ballance, P. J. Gawthrop, J. Gribble, and J. O'Reilly, "A nonlinear disturbance observer for two link robotic manipulators," Proceedings of the 38th IEEE Conference on Decision and Control, vol. 4, pp. 3410-3415, 1999.

[24] L. Acho and F. Pozo, "Sliding mode control of hysteretic structural systems," International Journal of Innovative Computing, Information and Control, vol. 5, no. 4, pp. 1081-1087, 2009. 